\title{
NERI PROJECT 99-119. "A NEW PARADIGM FOR AUTOMATIC DEVELOPMENT OF HIGHLY RELIABLE CONTROL ARCHITECTURES FOR NUCLEAR POWER PLANTS." PHASE-2 PROGRESS REPORT
}

J. March-Leuba

J. A. Mullens

R. T. Wood

R.A. Kisner

Oak Ridge National Laboratory

B. R. Upadhyaya

The University of Tennessee, Knoxville

J. M. Doster

C. W. Mayo

North Carolina State University

September 2001

Prepared by the Oak Ridge National Laboratory Oak Ridge, Tennessee 37831

Managed by

UT-Battelle, LLC

For

U.S. Department of Energy

Under contract DE-AC05-00OR22725 


\section{DISCLAIMER}

This report was prepared as an account of work sponsored by an agency of the United States government. Neither the United States Government nor any agency thereof, nor any of their employees, makes any warranty, express or implied, or assumes any legal liability or responsibility for the accuracy, completeness, or usefulness of any information, apparatus, product, or process disclosed, or represents that its use would not infringe privately owned rights. Reference herein to any specific commercial product, process, or service by trade name, trademark, manufacturer, or otherwise, does not necessarily constitute or imply its endorsement, recommendation, or favoring by the United States Government or any agency thereof. The views and opinions of authors expressed herein do not necessarily state or reflect those of the United States Government or any agency thereof. 


\section{TABLE OF CONTENTS}

Acknowledgment ………........................................................................................

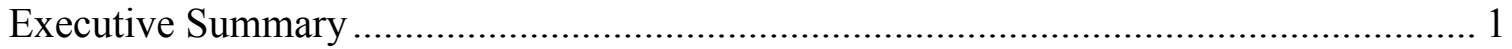

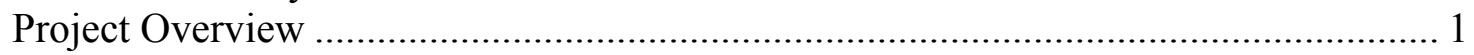

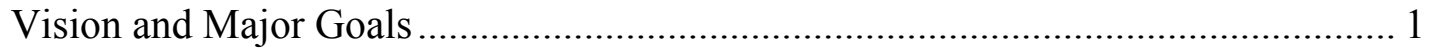

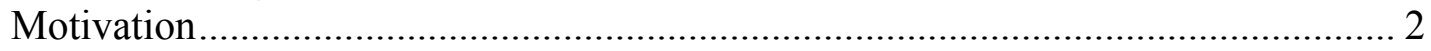

Program Organization................................................................................... 2

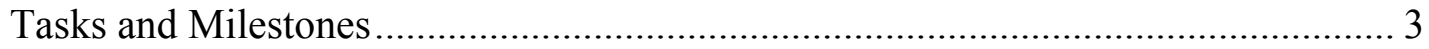

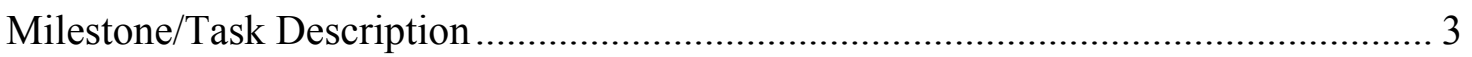

Milestone/Task Description...................................................................................... 3

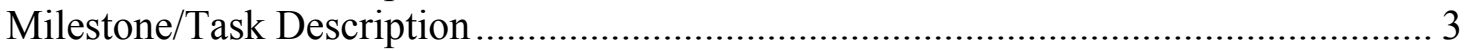

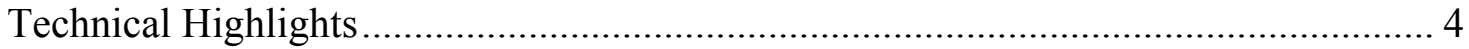

Task 1. Advanced Control Tools and Methods ....................................................... 4

Task 1.1 Adaptive Inverse Control Algorithms ....................................................... 4

Task 1.2 Control-Priority Mode Selection Algorithms........................................... 4

Task 1.3 Controllers for Feedwater Systems ....................................................... 5

Task 1.4 Requirements-Driven Control System Design ......................................... 6

Task 2. Advanced Monitoring and Diagnostics..................................................... 8

Task 2.1 Data-Driven Prediction of Process Variables .......................................... 8

Task 2.2 Detection of Simultaneous Faults ........................................................ 9

Task 2.3 Implementation of On-line Diagnostics System ..................................... 9

Task 3. Nuclear Power Simulation and Reliability Methods.................................... 13

Task 3.1 Plant Model Refinement .................................................................... 13

Task 3.2 Reliability Analysis Methods Development ........................................... 13

Task 4. Nuclear Information System Architecture and Integration .......................... 14

Task 4.1 Requirements for Information-System Architecture............................... 14

Task 4.2 Application Programming Interfaces ................................................ 14

Task 4.3 Application Environment ............................................................... 15

Task 5. Project Integration............................................................................. 15

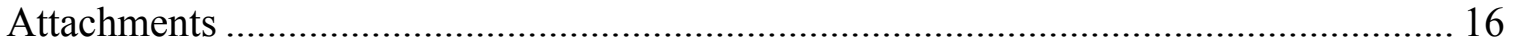

Attachment I. Task 1 - Advanced Control Tools and Methods ...................................... 16

Attachment II. Task 2 - Advanced Monitoring and Diagnostics ................................... 16

Attachment III. Task 3 - Nuclear Power Simulation and Reliability Methods............. 16

Attachment IV. Task 4 - Nuclear Information System Architecture ............................ 16

Attachment V. Publication List ................................................................................. 16 


\section{Acknowledgment}

This research has been conducted under a grant funded by the U.S. Department of Energy Nuclear Energy Research Initiative (NERI,) project number NERI 99-119. The first page of this report lists as authors the principal investigators from the three organizations. A detailed list of all major contributors follows
A.M.H. Basher (SCSU)
C. R. Brittain (ORNL)
W. Dong (NCSU)
J. M. Doster (NCSU)
N. Kaista (UTK)
R. A. Kisner (ORNL)
B. Lu (UTK)
J. March-Leuba (ORNL)
J. A. Mullens (ORNL)
C. W. Mayo (NCSU)
A. Sakabe (NCSU)
B. R. Upadyahya (UTK)
R. T. Wood (ORNL)
K. Zhao (UTK) 


\section{Executive Summary}

This report describes the tasks performed and the progress made during Phase 2 of the DOE-NERI project number 99-119 entitled Automatic Development of Highly Reliable Control Architecture for Future Nuclear Power Plants. This project is a collaboration effort between the Oak Ridge National Laboratory (ORNL,) The University of Tennessee, Knoxville (UTK) and the North Carolina State University (NCSU). ORNL is the lead organization and is responsible for the coordination and integration of all work.

\section{Project Overview}

\section{Vision and Major Goals}

This research focuses on the development of methods for automated generation of control systems that can be traced directly to the design requirements for the life of the plant.

Our final goal is to "capture" the design requirements inside a "control engine" during the design phase. This control engine is, then, not only capable of designing automatically the initial implementation of the control system, but it also can confirm that the original design requirements are still met during the life of the plant as conditions change.

This control engine captures the high-level requirements and stress factors that the control system must survive (e.g. a list of transients, or a requirement to withstand a single failure.) The control engine, then, is able to generate automatically the controlsystem algorithms and parameters that optimize a design goal and satisfy all requirements. As conditions change during the life of the plant (e.g. component degradation, or subsystem failures) the control engine automatically "flags" that a requirement is not satisfied, and it can even suggest a modified configuration that would

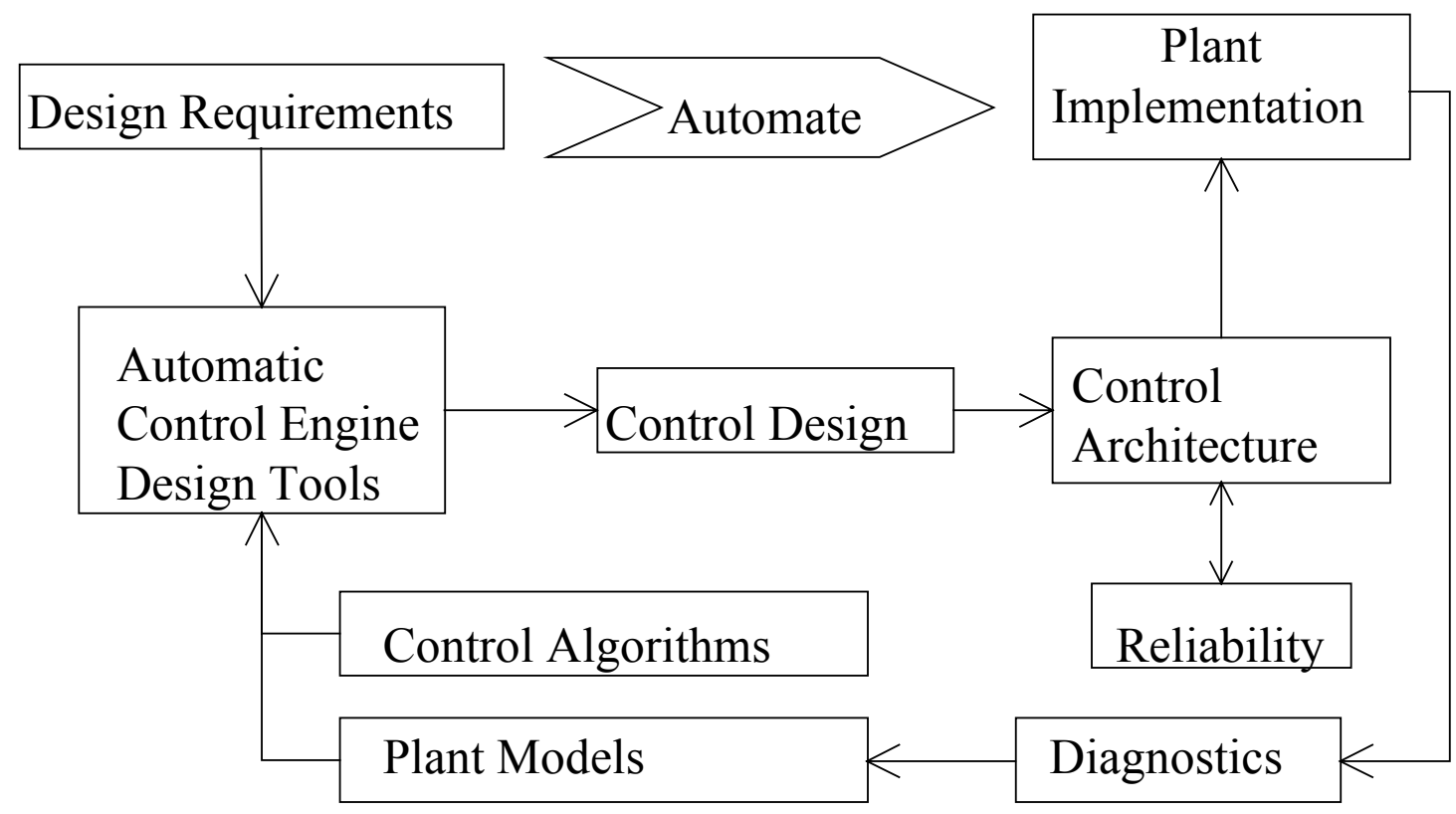

Figure 1. Schematic diagram of the automated control design process 
satisfy it. This control engine concept is shown schematically in Fig. 1.

The implementation of this "control-engine" design methodology requires the following steps, which are described in detail in the attachments to this report:

1. Selection of Design Requirements Related to Control System Performance

2. Implementation of Requirements in Mathematical Form

3. Development of a Control Algorithm Library

4. Development and Validation of Plant Models

5. Automated Control Design Development

6. Development of Control Architectures

7. Control Design Implementation

8. Development of Diagnostics Methods to Update the Plant Model

\section{Motivation}

In the past two decades there have been significant progress in the automated development of control algorithms and on computer aids for fast and reliable implementation of those algorithms. Indeed, there are a number of commercial off-theshelf software packages that can help design and parameterize control systems. Some of these packages even have graphical interfaces, which facilitate the use of alreadyvalidated software modules. It is not the goal of this research to reproduce this existing work

The purpose of this research is to extend the current state of the art, so that the design requirements are captured in a control engine, which is used during the life of the facility to confirm that the original requirements are still met as plant conditions change. Capturing these requirements is of special relevance to the nuclear industry, where the plant life is often 40 to 60 years, and the system requirements are not always obvious.

Nuclear plant availability has improved significantly in the past decade. One reason for this improvement is that plants are relying more heavily on the control system response to prevent unnecessary scrams. Thus, improved economic operation of the plant relies more and more on these "hidden" requirements, where the control system is quite capable of maintaining steady state or normal operation, but cannot handle a postulated transient because of a degraded condition. By capturing the requirements into the control system itself, we can make the operator aware of this deficiency, so that either the degraded condition can be corrected in time, or the control system updated to handle it.

\section{Program Organization}

This project is a collaborative effort between the Oak Ridge National Laboratory (ORNL), The University of Tennessee, Knoxville (UTK), and the North Carolina State University (NCSU). ORNL is the lead organization and is responsible for the coordination and integration of all work. The program is organized into five major tasks, which address the major steps described above.

1. Advanced Control Tools and Methods. ORNL is the responsible organization. 
2. Advanced Monitoring and Diagnostics. UTK is the responsible organization.

3. Nuclear Power Simulation and Reliability Methods. NCSU is the responsible organization.

4. Nuclear Information System Architecture and Integration. ORNL is the responsible organization.

5. Project Integration. ORNL is the responsible organization.

\section{Tasks and Milestones}

All tasks and milestones for Phase 1 have been completed and detailed reports on each of them are attached to this summary. The following tables shows a listing of all tasks for each of the three project phases along with the responsible organization and planned completion dates.

Phase 1:

\begin{tabular}{|l|l|l|l|}
\hline \multirow{2}{*}{ Milestone/Task Description } & \multirow{2}{*}{ Org. } & \multicolumn{2}{|c|}{ Completion Date } \\
\cline { 3 - 4 } & & Planned & Actual \\
\hline 1.1 Adaptive Inverse Control Algorithms & ORNL & $6 / 00$ & $6 / 00$ \\
\hline 1.2 Control-Priority Mode Selection Algorithms & ORNL & $9 / 00$ & $7 / 00$ \\
\hline 2.1 Data-Driven Prediction of Process Variables & UTK & $6 / 00$ & $6 / 00$ \\
\hline 2.2 Detection of Simultaneous Faults & UTK & $9 / 00$ & $7 / 00$ \\
\hline 3.1 Plant Model Refinement & NCSU & $9 / 00$ & $8 / 00$ \\
\hline 4.1 Requirements for Information-System Architecture & ORNL & $9 / 00$ & $7 / 00$ \\
\hline 5.1 Development of Integrated Software & ORNL & $9 / 00$ & $7 / 00$ \\
\hline
\end{tabular}

Phase 2:

\begin{tabular}{|l|l|l|l|}
\multirow{2}{*}{ Milestone/Task Description } & \multirow{2}{*}{ Org. } & \multicolumn{2}{|c|}{ Completion Date } \\
\cline { 3 - 4 } & & Planned & Actual \\
\hline 1.3 Controllers for Feedwater Systems & ORNL & $9 / 01$ & $7 / 01$ \\
\hline 1.4 Requirements-Driven Control System Design & ORNL & $6 / 02$ & Phase 3 \\
\hline 2.3 Implementation of On-line Diagnostics System & UTK & $9 / 01$ & $8 / 01$ \\
\hline 3.2 Reliability Analysis Methods Development & NCSU & $9 / 01$ & $8 / 01$ \\
\hline 3.3 Reliability Analysis Methods Integration & NCSU & $6 / 02$ & Phase 3 \\
\hline 4.2 Application Programming Interfaces & ORNL & $6 / 01$ & $6 / 01$ \\
\hline 4.3 Application Environment & ORNL & $9 / 01$ & $8 / 01$ \\
\hline 5.1 Development of Integrated Software & ORNL & $9 / 01$ & $8 / 01$ \\
\hline
\end{tabular}

Phase 3:

\begin{tabular}{|l|l|l|l|}
\hline & \multirow{2}{*}{ Org. } & \multicolumn{2}{|c|}{ Completion Date } \\
\cline { 3 - 4 } Milestone/Task Description & & Planned & Actual \\
\hline 1.4 Requirements-Driven Control System Design & ORNL & $6 / 02$ & \\
\hline 2.4 Requirements for Implementation on FDI System & UTK & $9 / 02$ & \\
\hline 3.3 Reliability Analysis Methods Integration & NCSU & $6 / 02$ & \\
\hline 4.4 Integrated Applications & ORNL & $9 / 02$ & \\
\hline 5.1 Development of Integrated Software & ORNL & $9 / 02$ & \\
\hline
\end{tabular}




\section{Technical Highlights}

\section{Task 1. Advanced Control Tools and Methods}

The ultimate goal of this task is to develop and demonstrate the control-engine concept. To this end, we have developed libraries of control algorithms, and we have successfully applied some of them to a prototype control engine problem.

\section{Task 1.1 Adaptive Inverse Control Algorithms}

This task was completed $100 \%$ under Phase 1 of this project. Under this task, we developed a general methodology for handling the sensor and actuator nonlinearities as piecewise linear functions. The piecewise linear functions provide very good approximations to characteristics such as hysteresis, dead zone, and backlash. The piecewise functions have sufficient number of free parameters to permit a realistic representation of most physical situations that occur with valves, rod controls, and other types of devices that occur in power plant control loops. The adaptive iteration updates the parameter estimates in the inverse law. Since such parameters are usually not known and can change with component wear and operating conditions, the advantage of the adaptive inverse control strategy is that it can adjust automatically using operating data. This type of algorithm eliminates the need for information from higher levels of the control algorithm to handle the performance characteristics. We have implemented inverse control algorithms for different non-linear actuator problems, such as dead-zone compensation or backslash non-linearity. The details are shown in Attachment I of the Phase I report.

Figures 2 through 4 show an example application of this technique to an actuator with a dead zone. As seen in Figure 4, the inverse control algorithm removes completely the non-linearity and allows for a simpler and more robust control system design.

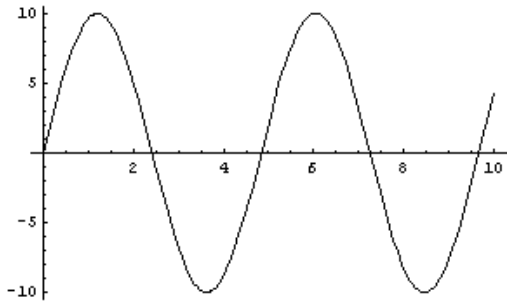

Figure 2. Input signal to actuator.

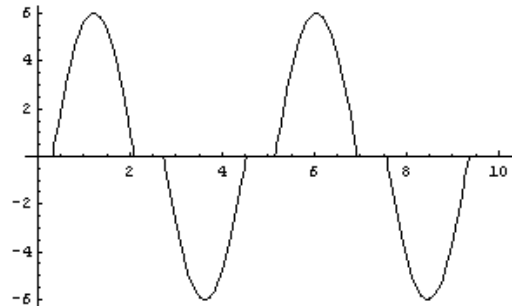

Figure 3. Output signal from nonlinear actuator with dead zone.

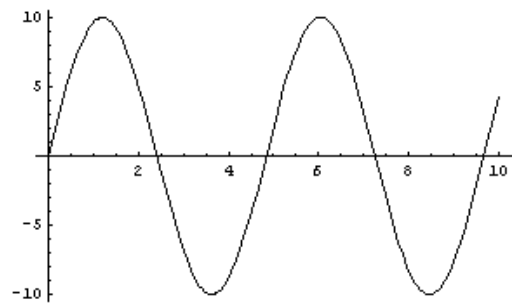

Figure 4. Output signal from nonlinear actuator with dead zone compensation.

\section{Task 1.2 Control-Priority Mode Selection Algorithms}

This task was completed $100 \%$ under Phase 1 of this project. Control priority mode selection is a strategy that allows autonomous agents to select the best control mode for a given condition. The control system designer provides detailed information needed to select the highest priority regulation objectives required to maintain the controllability of the full system for different failure states of actuators. For example, when one actuator saturates or is placed in manual, that actuator no longer responds to automatic control 
signals. The controllability of the full system is reduced in rank by one degree due to the loss of one actuator. The highest regulation objective is changed to maintain the most important objective in response to a reduced controllability rank of the full system.

Control priority mode selection has been implemented using the "socket" algorithms described in the Phase-1 Report. These control sockets use information from several sources to select the optimal algorithm given the current circumstances based on the entropy value calculated for each option. These sources of information include the online diagnostic systems, a library of available control algorithms, and repair and maintenance information. Proof of principle of this algorithm has been successfully demonstrated using a nine-component system with two possible control algorithms.

\section{Task 1.3 Controllers for Feedwater Systems}

Under this task, optimal feedwater controllers have been developed for a prototypical Utube steam generator. Full details are provided in Attachment I of this report. Figure 5 shows one such controller. This is a typical three-element controller, where the steam generator level is controlled by setting up a feedback loop on the measured water level

$$
\begin{aligned}
& \Delta F C V=k_{p}(P) \times\left[\begin{array}{l}
\left(L-L_{\text {set }}\right)+ \\
k_{3}(P) \times\left(w_{l}-w_{s}\right)
\end{array}\right] \\
& +k_{i}(P) \times \int\left(L-L_{\text {set }}\right) d t
\end{aligned}
$$
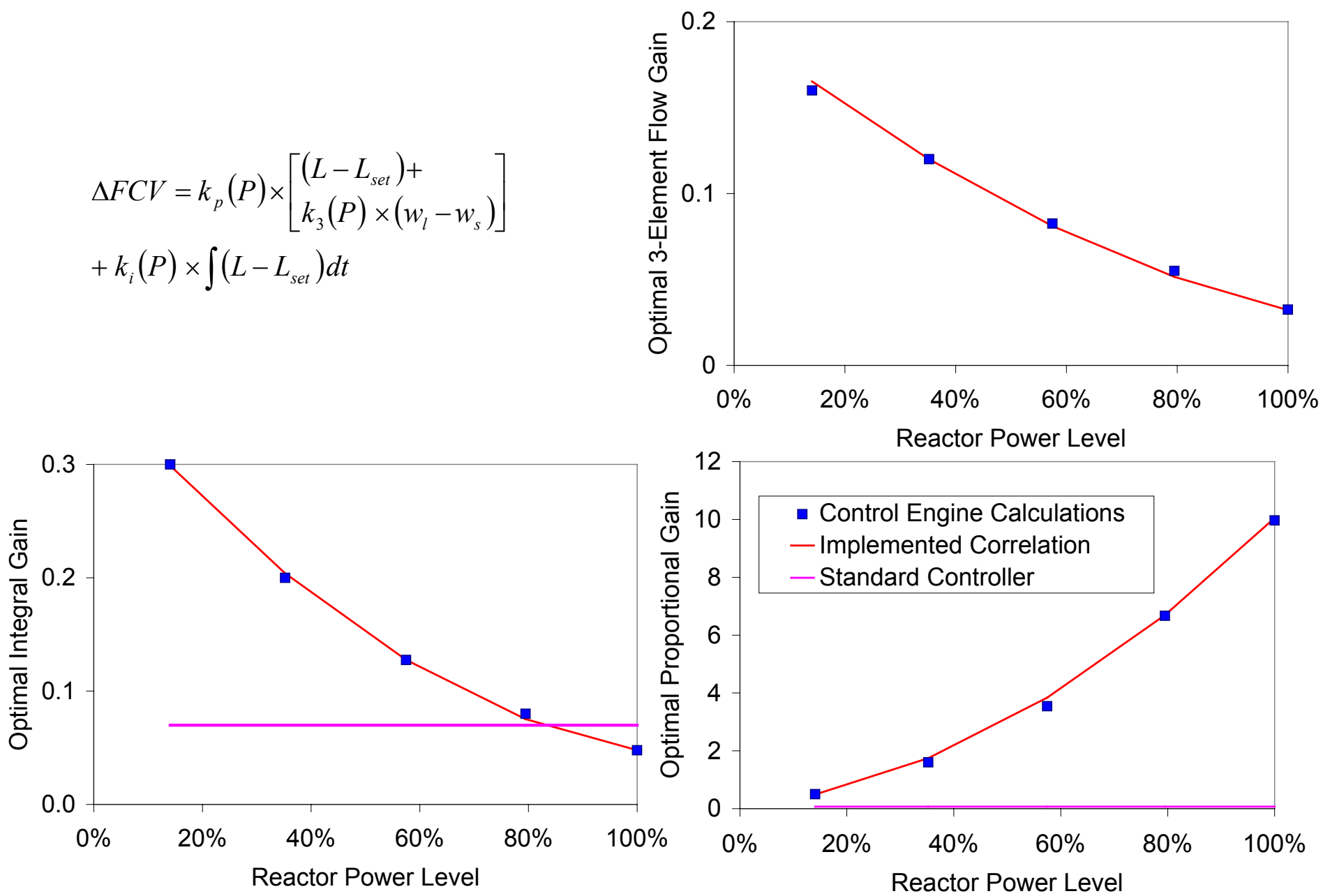

Figure 5. Power-Dependent Optimal Controller 
plus the mismatch between the steam flow and feedwater flows. By adding this flow mismatch in the three-element logic, these types of controllers can handle the well-known shrink and swell phenomenon, where the two-phase water level temporarily drops when additional cold feedwater is injected because it collapses steam voids.

Steam generator dynamics are non-linear and change significantly with operating power level as the void fraction changes. To compensate for those changes, the controller shown in Figure 5 was designed with power-dependent gains. In this way, the controller can be designed to be significantly more "aggressive" and have better performance at full power without losing stability at low power levels. As seen in Figure 5, our controller design provides a tradeoff between proportional, integral, and three-element gains as power changes. This results in greatly improved performance, especially at high powers where the steam generator operates most of the time.

What makes this controller unique, however, is not its performance characteristics, but the fact that it was designed completely automatically using the design engine of Task 1.4. In essence, we have captured the design requirements for this steam generator in the control engine and a new optimized controller can be calculated at any time in the life of the facility if conditions or equipment changes (e.g., component failures or replacements) occur.

\section{Task 1.4 Requirements-Driven Control System Design}

In the past two decades there has been significant progress in the automated development of control algorithms and on computer aids for fast and reliable implementation of those algorithms. Indeed, there are a number of commercial off-the-shelf software packages that can help design and parameterize control systems. Some of these packages have graphical interfaces, which facilitate the use of already-validated software modules. It is not the goal of this research to reproduce this existing work. The purpose of this task is to extend the current state of the art, so that the performance requirements are captured in a Control Engine, which is used during the life of the facility to confirm that the original requirements are still met as plant conditions change. Capturing these requirements is of special relevance to the nuclear industry, where the plant life is often 40 to 60 years, and the system requirements are not always obvious.

In our proposed methodology, we achieve this goal by reformulating the performance requirements as mathematical constraints of a minimization problem. For example, one such constraint could be that the steam generator control system must survive an anticipated over-cooling event without scram. The Control Engine runs in the background in supervisory mode and continuously evaluates whether these constraints are satisfied given the current state of the plant. If they are not, it starts an iterative minimization calculation that suggests to the operator optimal control parameter settings or even different control strategies if the current one is inadequate. Since changes to the plant over its 40 to 60 year life are slow in nature, we do not envision the Control Engine running in a closed loop, and automatically changing control parameters or strategies. Its function is more of an advisory nature by producing some kind of an alarm when the original control-system performance-requirements are not satisfied under the present 
conditions (e.g., hardware failures, or plant reconfiguration.) In addition to the alarm, the Control Engine can also suggest new control system settings that would satisfy the performance requirements under the present plant condition.

For this demonstration, we have developed Control Engine prototype software using standard off-the-shelf minimization algorithms and we have coupled it to several simulation programs. For the application, we have chosen a complex, high-fidelity PWR simulator developed in Task 3.1. This PWR simulator is a large Fortran code, which we have coupled to the control engine without modification; thus demonstrating that this technique can be applied to essentially any engineering simulator. For this example, the performance requirements are defined as avoiding scram for: (1) a 10\% power reduction, and (2) a 40 degrees $\mathrm{F}$ reduction in feed-water temperature; these are arbitrary requirements and other may have been chosen.

The results of the Control Engine optimization for the two above transients are shown in Figure 6 and Figure 7; which show the steam generator level during the simulated transient with the original control parameter settings and with the optimized parameters. The thermal power (i.e., steam flow to the turbine) is controlled very accurately during the transient, and we did not observe any unusual neutron-flux power oscillations in the reactor core. These results are obtained by iterating using the simulator with different control parameters and choosing those parameters that minimize the overall error for both transients. Note that by using this minimization technique, we do not require to linearize or Laplace-transform the reactor model. This provides us with two relevant features: (1) we can use existing complex models "as is", and (2) non-linear or non-minimum phase phenomena, such as the well-known shrink and swell effect, are inherently taken into account.

In addition to the ability of generating control algorithms and parameters automatically, the Control Engine captures the performance requirements that are expected of the control system from the life of the facility. This allows for on-line reconfiguration of the control system as equipment degrades, or when changes occur in the facility.

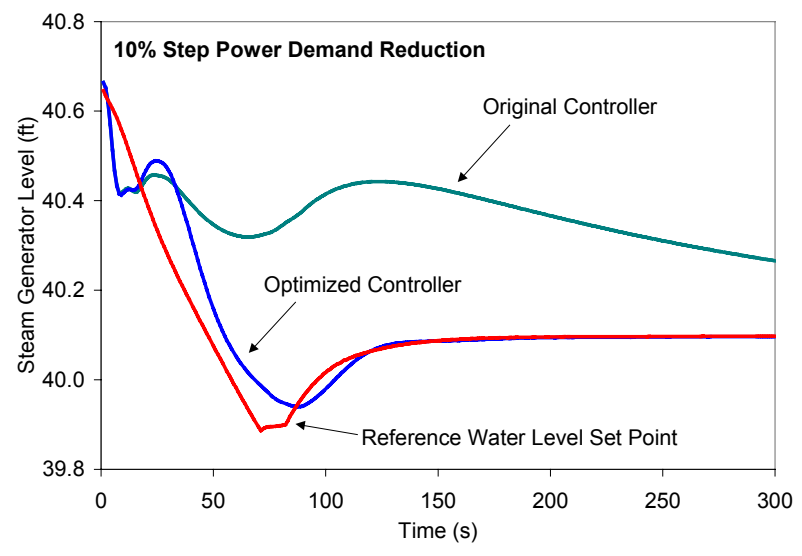

Figure 6. The Control Engine automatically calculates the level control strategy that satisfy all of the performance requirements

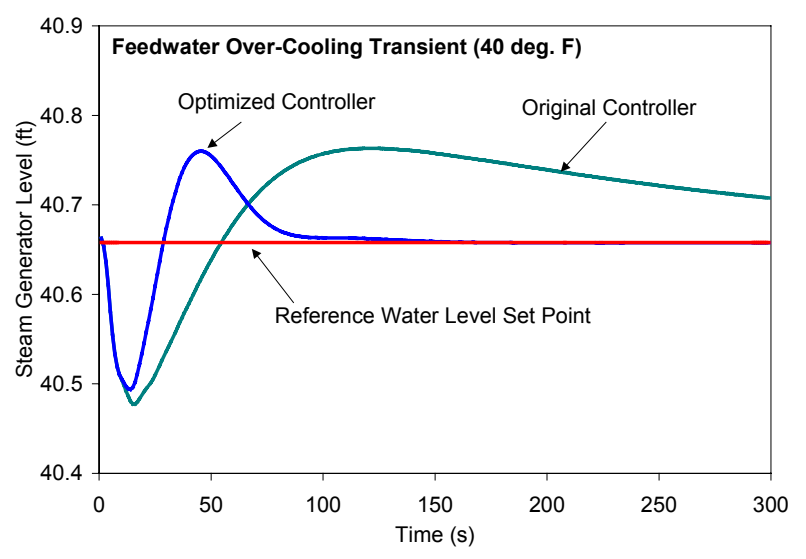

Figure 7. The Control Engine calculates the optimal control strategy for multiple postulated transients and performance requirements 


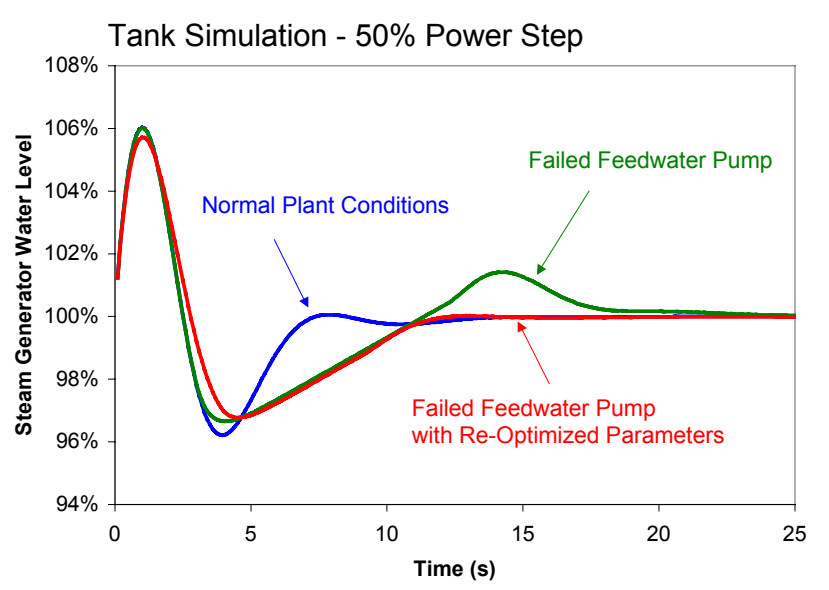

Figure 8. Example of Control Engine decision following a failed feedwater pump. Control parameters are re-optimized to minimize overshoot for a postulated power step transient

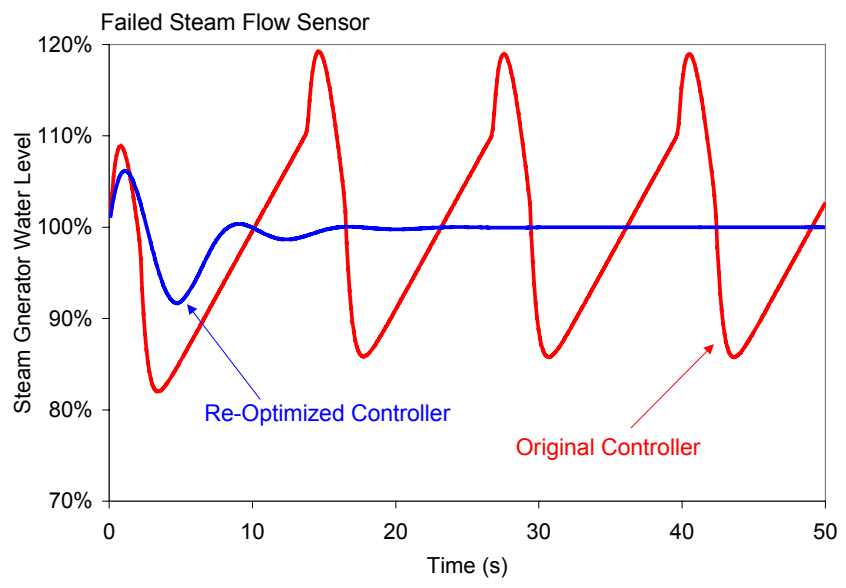

Figure 9. Example of Control Engine decision after diagnosis of an incipient failure of the steam flow sensor. Control parameters are reoptimized to prevent instability

Figure 8and Figure 9show examples of this feature. In the example of Figure 8, a feedwater pump is assumed failed, so that the maximum feedwater flow available to the controller is limited. The Control Engine automatically recognizes that, because of the maximum flow limitation, the original controller will produce a significant overshoot. The Control engine automatically suggests a new set of control parameters that are less aggressive and minimize the overshoot caused by the new plant condition. In the example of Figure 9, the diagnostics system has detected an incipient failure of a steam flow sensor. Then Control engine, then, evaluates the impact of this failure (by assuming completely failed) and concludes that if the sensor fails, the controller will become unstable. It then, automatically, re-computes optimizes control parameters that do not use the steam flow sensor, and it suggests a re-optimized controller that will not be sensitive to the sensor failure should it occur. Note that this new controller is not as good as the original controller, but it is more fault-tolerant given the information we now know about the probability of failure of the sensor.

\section{Task 2. Advanced Monitoring and Diagnostics}

The objective of this research task is to develop an on-line monitoring system for fault detection and isolation (FDI) of sensors and field devices in a nuclear power plant. In this research emphasis has been given to process instrumentation in a nuclear power plant such as temperature, pressure, flow, level transmitters, and measurements of control functions. Field devices include valve actuators, control modules, spray and heater systems, pumps, and other similar equipment. The goal of this task is to provide diagnostics information to a system executive for enhanced decision-making by the plant control system.

\section{Task 2.1 Data-Driven Prediction of Process Variables}

This task was completed $100 \%$ during Phase 2 of this project. Under this task we completed the development of data-driven models for the characterization of sub-system 
dynamics for predicting state variables, control functions, and expected control actions. We have also developed the Principal Component Analysis (PCA) approach for mapping system measurements, and a nonlinear system modeling approach called the Group Method of Data Handling (GMDH) with rational functions, and includes temporal data information for transient characterization..

\section{Task 2.2 Detection of Simultaneous Faults}

This task was completed $100 \%$ during Phase 2 of this project. Under this task, we completed the development of a fault detection and isolation module that combines system operational knowledge (including system simulation) and a rule-based logic for $F D I$ of both single and dual faults in dissimilar sensor and field devices. In addition, we have developed a complimentary approach that quantifies the prediction errors using a fault pattern classification technique.

The above techniques have been applied to a laboratory process control loop using both simulation and actual loop measurements. The techniques have been demonstrated for detecting and isolating faults in sensors and devices in a U-tube steam generator (UTSG) in a pressurized water reactor (PWR) using a full-scope PWR simulator developed by North Carolina State University. The application to the laboratory system and preliminary application to a PWR steam generator were described in the Phase 1 Report.

\section{Task 2.3 Implementation of On-line Diagnostics System}

The following major milestones were accomplished during Phase 2:

- Development and testing of time-dependent $\boldsymbol{G M D H}$ modeling module for state and control function prediction.

- Development and testing of linear and non-linear $\boldsymbol{P C A}$ method for system characterization.

- Generation of an extensive simulation database for normal and fault operation for a 1,300 MWe PWR plant in the power range $20 \%-100 \%$.

- Demonstration of the FDI module for both single and dual/simultaneous faults and includes the following highlights:

> Rule-based decision making.

> Fault isolation using fault residuals and pattern classification.

$>$ Steady state and transient plant operation conditions.

$>$ Combination of sensors and valve actuators.

> Use of both Simulink ${ }^{\mathrm{TM}}$ and NCSU-PWR simulation codes.

> Demonstration of the functional features of the FDI module using the Halden PICASSO platform.

Figure 10 shows the schematic of a typical UTSG with various measurements. 


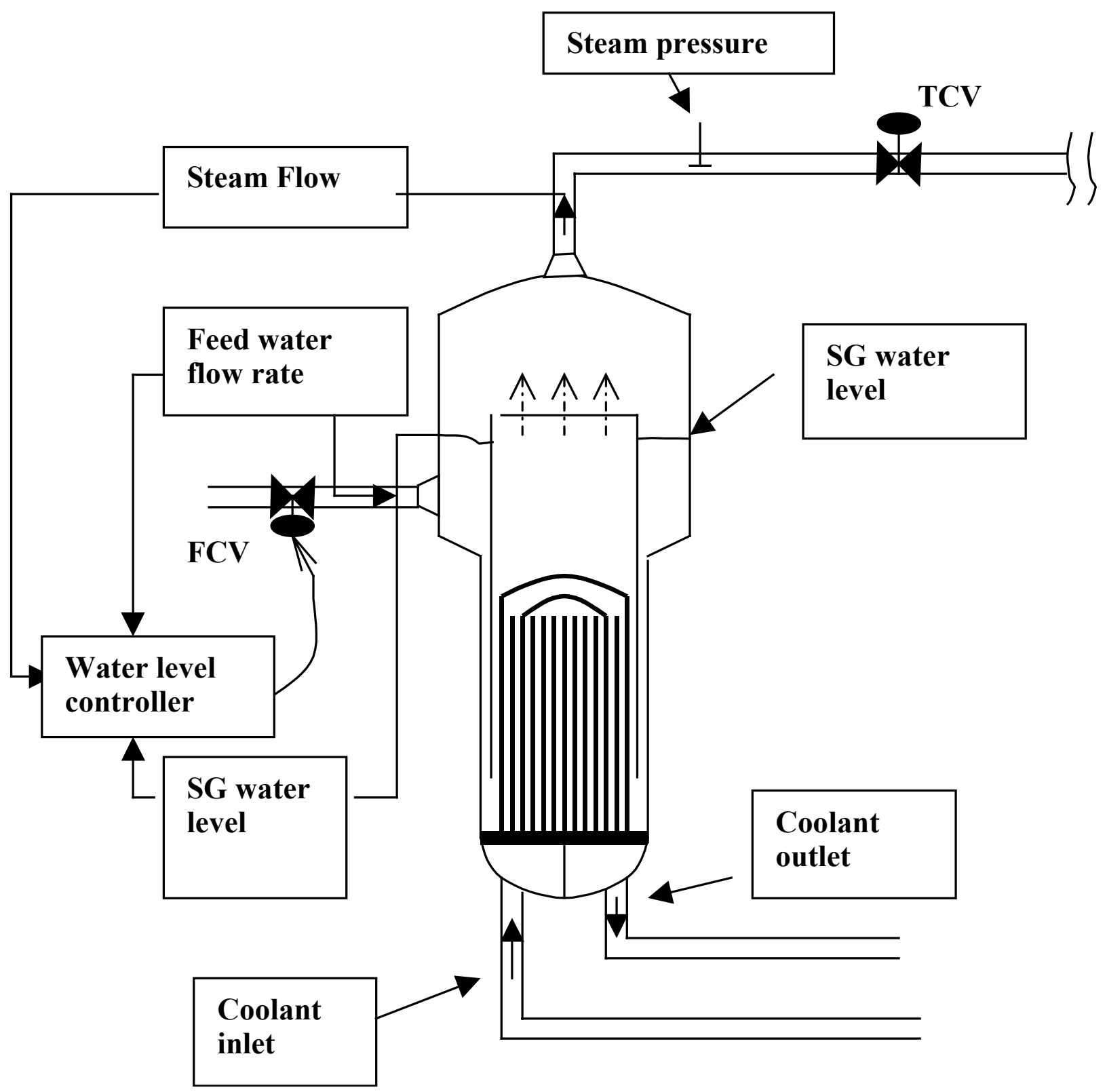

Figure 10. Schematic of a typical UTSG system showing various measurements 
A comparison of the training data and prediction using the $\boldsymbol{G M D H}$ modeling of feed control valve (FCV) is given in Figure 11. This model for the detection of single fault has the form (TCV: Turbine Control Valve)

FCV Position $=\mathrm{f}\left(\right.$ Reactor power, Steam flow rate, $\mathrm{SG}$ water level, $\mathrm{TCV}_{1}$ position $)$.

Similar models are developed for other related variables. The residual pattern of signals due to FCV dead band change is plotted in Figure 12. The pattern in these residuals is an indication of the type of fault and this knowledge is used for fault isolation.
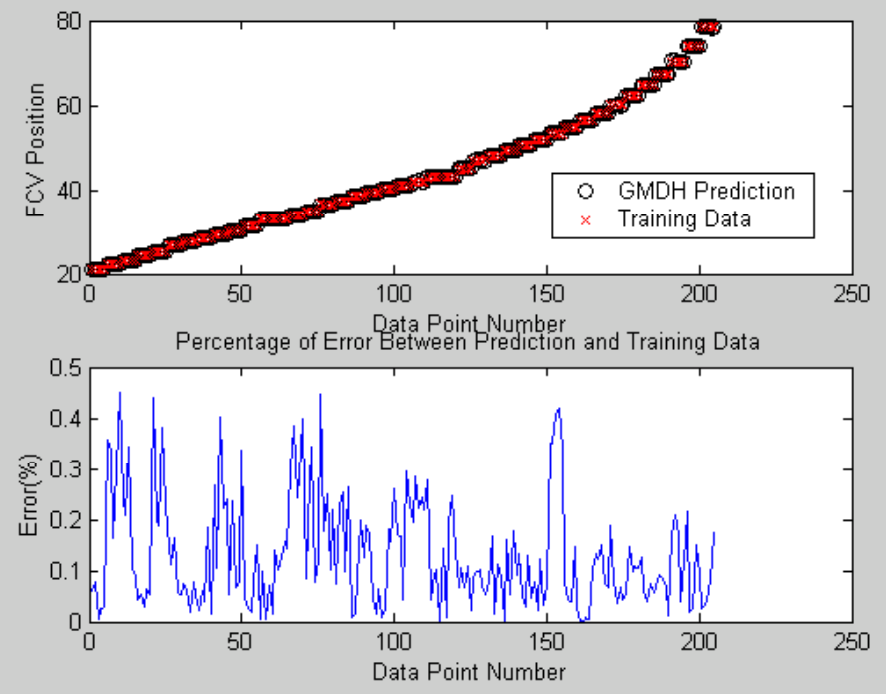

Figure 11. Static GMDH model training results for feed control valve (FCV) position

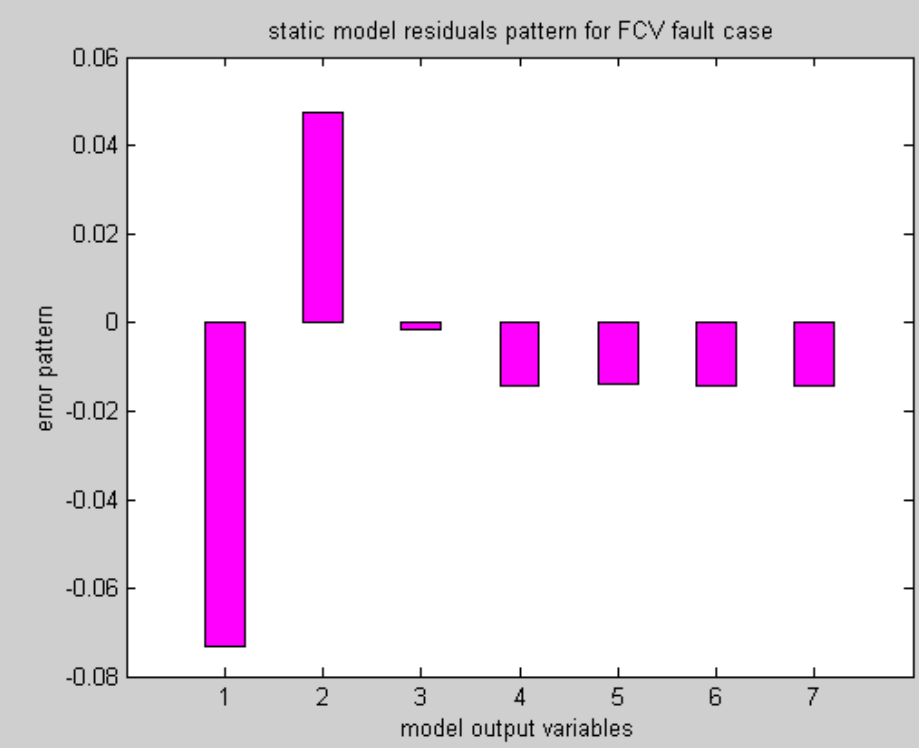

Figure 12. Pattern of prediction errors of seven variables for the case of FCV fault 
An example of tracking device faults during a plant transient is illustrated in Figure 13. The figure shows the actual and predicted values of the TCV flow rate for the case of TCV time constant degradation. Note that the prediction error changes with time, and is typical of patterns of faults during a plant transient.

The detection and isolation of dual faults requires the development of multiple models and a knowledge base that describes the consistency of the models. Predictive models of FCV flow rate, steam flow rate, SG pressure, FCV position, SG level and SG level controller output were developed using Adaptive Network-based Fuzzy Inference System (ANFIS). These models were then used to track their predictability for various fault types (six single and ten dual faults). All the enumerated faults were detected. A table showing the consistency checking of the ANFIS models is given in the section on Fault Detection and Isolation of Plant Sensors and Field Devices.
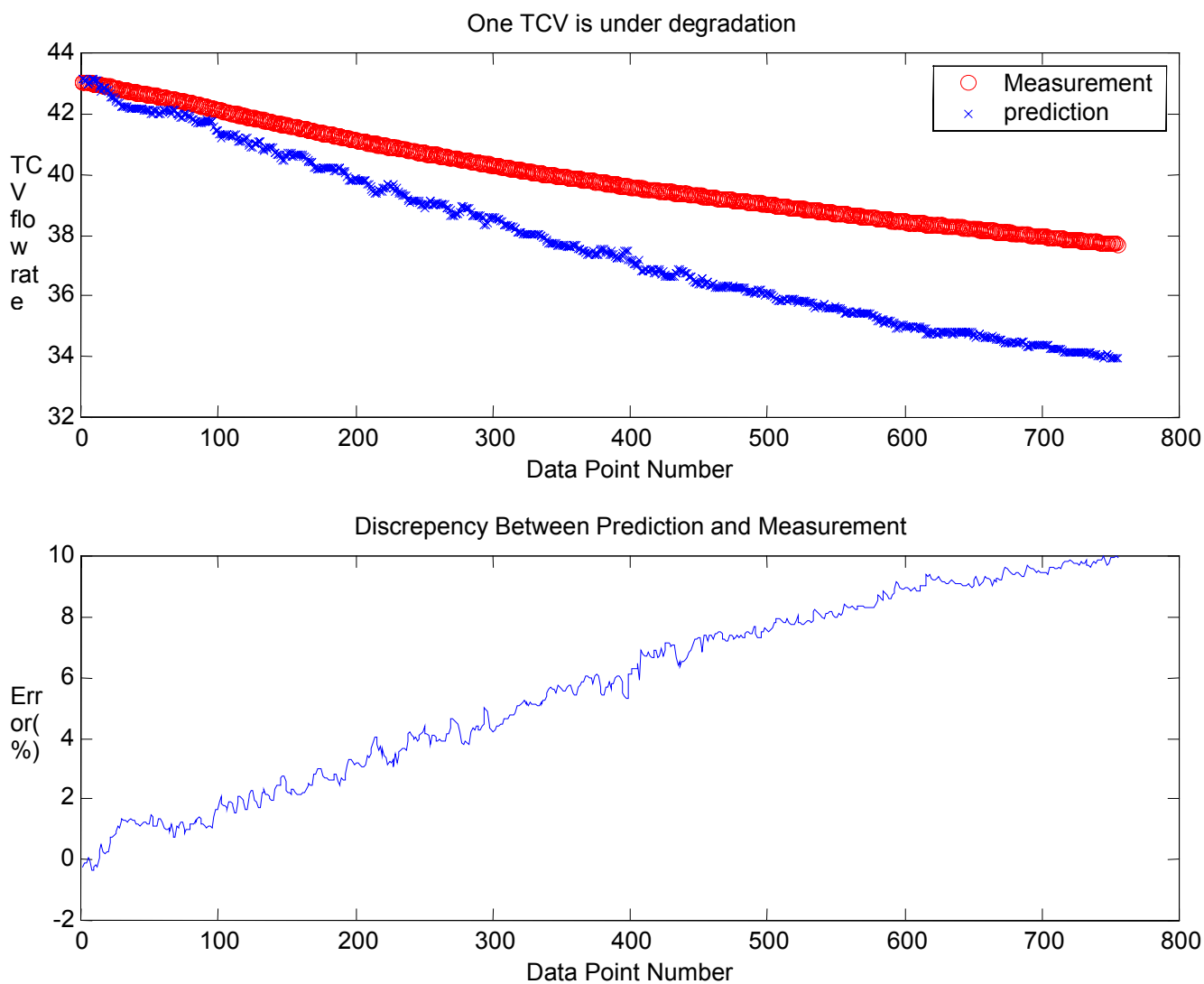

Figure 13. Turbine control valve (TCV) flow rate showing the actual and predicted values 


\section{Task 3. Nuclear Power Simulation and Reliability Methods}

\section{Task 3.1 Plant Model Refinement}

This task was completed $100 \%$ during Phase 1 of this project. Since 1996, a full plant engineering simulation code has been under development and in use at North Carolina State University for simulating the dynamic response of pressurized water reactors during normal operational transients as well as design basis events, with the exception of LOCA. The model is currently capable of simulating multi-loop PWR systems with U-Tube steam generators including asymmetric loop operation. Both primary and secondary sides are represented, including balance of plant and control systems. Plant protection systems are modeled including the normal spectrum of reactor trips and engineered safety features.

The work under this task required improvements in the plant simulator, which consist of refinements to existing models as well as development of new models to support the complete secondary side modeling effort. The secondary side has been broken into three separate subsystems. The first subsystem consists of the main steam system from the steam generators to the turbine and includes the main steam safety valves, steam dump system and the turbine bypass system. In addition, refinements to existing models have been oriented toward improved component or sensor models and include 1) development of a new valve model which allows for arbitrary dead band (hysterisis) and time constant, 2) a general level sensor $(\Delta \mathrm{P})$ model which allows for the prediction of both true and indicated water level and 3) a RTD model for the prediction of fluid temperatures. Options have been added to the code to allow for degradation in the heat transfer across the steam generator from both fouling and blocked or plugged tubes, as well as the corruption of sensor outputs through step and ramp changes in sensor output with arbitrary levels of random noise.

\section{Task 3.2 Reliability Analysis Methods Development}

A strategy and methods have been defined to integrate and automate instrumentation and control system reliability analysis with nuclear power plant simulation. Input data is in the form of files that represent a list of all units that compose an I\&C system including the system identification, sensors, cables or other communication means, power sources, function units, and actuators. For each unit, input data consists of the type, tag number, power sources, applicable failure modes (high, low, on, of, open, closed, etc.), failure rates, linkage to all of the unit input sources, and linkage to all of the unit output destinations.

The reliability analysis functions will be incorporated into revised feedwater control system subroutines. Components considered will include sensors, cables, power sources, electronic function units, and actuators. The revisions will effectively implement a reliability block diagram model in the structure of the control algorithm so that each modeled unit in a system can be independently failed and then the effect propagated through the remaining units to the control output at each. Both a single failure dependent and a fault tolerant feedwater controller will be modeled so that differences between the two designs can be compared. 
Simulations will be run for each unit for and for each failure mode entered, and the plant control demands and process parameters will be automatically tested for changes. The results will be summarized in a Failure Modes and Effect report file that identifies the component, each failure mode tested, the associated failure rate, control demand change in response to the failure mode, and the change in key plant parameters such as steam pressure, steam generator level, feedwater flow, reactor power, primary pressure, and pressurizer level. These files can be subsequently printed or sorted by content by a data base manager.

The benefit of this simulation based reliability analysis is that it provides the ability to do both reliability calculations and failure modes and effects analysis in an automated manner. An additional benefit is that the simulation code can be used observe the plant response over periods of time that include plant process feedback effects. System design changes considered for mitigating or eliminating undesired responses can be tested.

\section{Task 4. Nuclear Information System Architecture and Integration}

The objective of this task to promote the development of an integrated control and information system architecture for future nuclear power plants that will provide the processing support for self-organizing control applications while isolating the algorithms themselves from hardware-dependent interactions. The research approach we are employing is to develop the concept of a Plant-Control Computing Environment (PCCE), identify functional requirements for such a computing environment, and demonstrate the PCCE concept. The PCCE demonstration will be accomplished through the development of key architectural components for use in a prototype application involving integrated balance-of-plant control and diagnostics functions.

\section{Task 4.1 Requirements for Information-System Architecture}

This task was completed $100 \%$ under Phase 1 of this project. This research addressed the control and information system architecture for future nuclear power plants involves the evolution of the PCCE concept and the generation of functional requirements. We generated functional requirements addressing: general design attributes, human-system interface requirements, control application interface requirements, computing platform interface requirements, monitoring and control requirements, fault handling and recovery requirements, system management requirements, and configuration requirements.

\section{Task 4.2 Application Programming Interfaces}

Part of the computing environment is a group of $\mathrm{C}++$ objects that mimic an instrument fieldbus. These objects act as instruments and controllers, through which signals flow between the plant simulation and the applications being developed. These applications access the plant simulator's internal variables through this link, giving them access to the simulated instrumentation and control elements of the plant. The flow of signals (digital data) through this network determines how the applications interact directly with the plant. The environment allows the applications to run on different computers and interact through the (real) Internet. 


\section{Task 4.3 Application Environment}

The Plant Computing Environment (PCE) is a distributed computing environment supporting a high-level supervisory control and monitoring system.

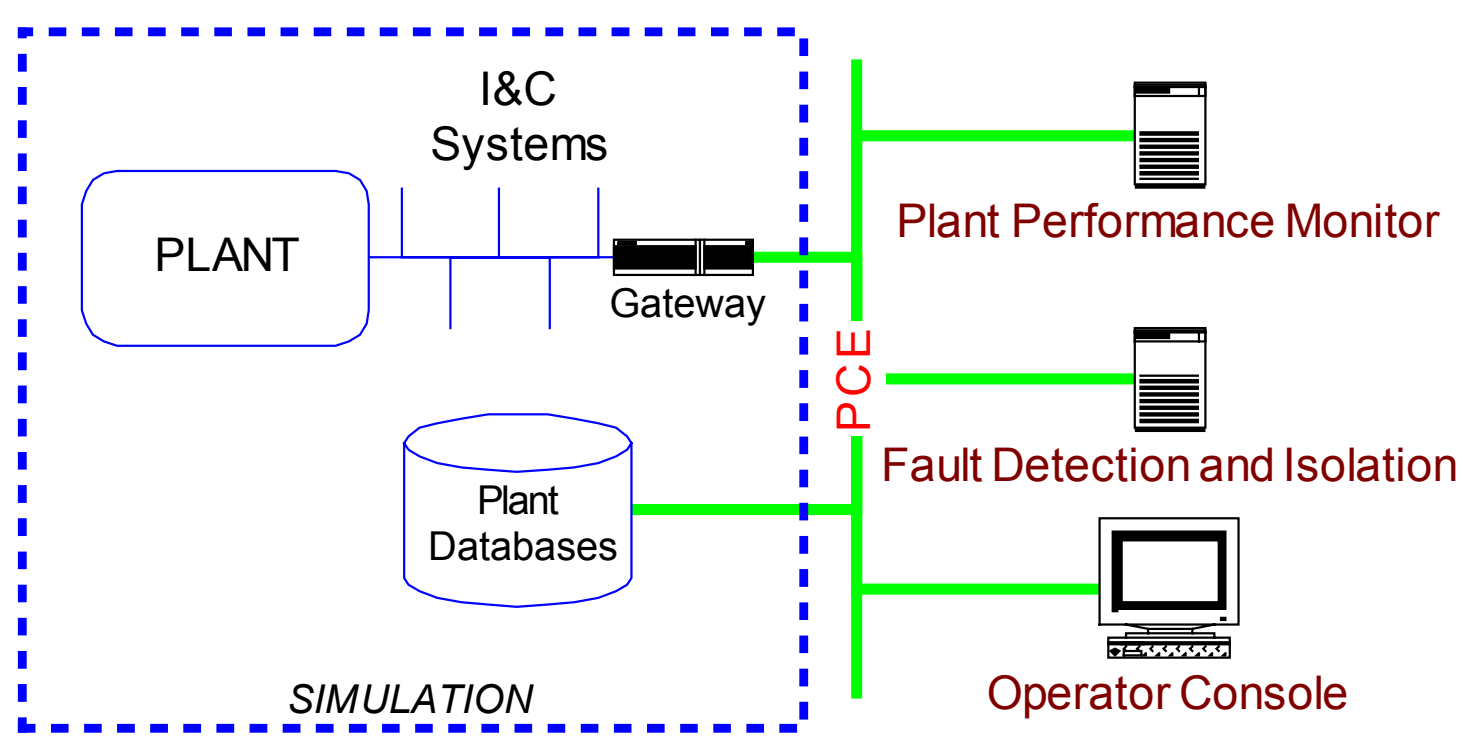

Figure 14 Plant Computing Environment and Applications.

The PCE provides communication and information services for the other applications of this project (Fault Detection and Isolation (FDI) application, Plant Performance Monitor, and Picasso display, and plant simulator). Part of this service is access to the simulated fieldbus environment described above, which carries the plant's dynamic data. The plant's static data, such as design information, is also available through the PCE Server. This design information is common to all applications, identifies all of the components of the plant, and can be the vocabulary of communications between them.

\section{Task 5. Project Integration}

This is mostly an administrative task of integration between the different participants: ORNL, UTK, and NCSU. The final objective of this task is to integrate modeling and diagnostics within the final control engine demonstration. 


\section{Attachments}

The following attachments are included

Attachment l. Task 1 - Advanced Control Tools and Methods

Attachment II. Task 2 - Advanced Monitoring and Diagnostics

Attachment III. Task 3 - Nuclear Power Simulation and Reliability Methods

Attachment IV. Task 4 - Nuclear Information System Architecture Attachment V. Publication List 